\title{
Modified Polyaxial S1 Screw Placement in Patients with Difficult Sacral Anatomy - Technical Report
}

\author{
Maziyar A. Kalani ${ }^{1}$, Gordon H. Li ${ }^{1}$, Stefan A. Mindea ${ }^{1}$, Jon Park ${ }^{2}$ \\ 1. Department of Neurosurgery, Stanford University School of Medicine 2. Department of Neurosurgery, \\ Stanford University Medical Center
}

$\square$ Corresponding author: Maziyar A. Kalani, mkalani@gmail.com

Disclosures can be found in Additional Information at the end of the article

\section{Abstract}

We present a case of a patient with unfavorable sacral angles and narrow iliac window created by large bilateral iliac crests who underwent L5-S1 anterior lumbar interbody fusion (ALIF) followed by posterior fixation using L5-pedicle screws and L5-S1-facet screws for lumbar degenerative disease. Due to the difficult sacral anatomy, S1 pedicle screw placement was deemed technically difficult. The use of a L5-S1 facet screw reduced the need for retraction and muscle dissection or inserting a pedicle screw via new incision. Although no longer the most commonly performed form of posterior instrumented fusion, facet screw fixation has similar pseudoarthrosis rates when compared to transpedicular screw fixation. Transfacet fixation, however, is less invasive compared to transpedicular fixation and can be incorporated with the traditional ALIF procedure. Moreover, facet screw fixation affords 4-cortical purchase of the facet joints and can be used in obese patients, patients with hypertrophic iliac crests and those with unfavorable sacral angles.

Categories: Neurosurgery

Keywords: medial-to-lateral fixation, alif, s1 screw, facet screw, degenerative disc disease, spinal fusion

\section{Introduction}

Transfacet screw fixation was initially introduced in the late 1940s by King and later modified by Boucher in the late 1950s as a method of spinal fixation through an ipsilateral approach [1, 2]. Magerl provided the most recent advance to transfacet fixation by describing a contralateral approach whereby a facet screw is entered in the contralateral lamina and passed through the ipsilateral facet joint [3]. This translaminar approach - although not a classical facet fixation was deemed more efficacious in immediate fixation but more technically challenging due to the

Received 12/03/2012 Review began 12/03/2012 Published 01/18/2013

\section{(c) Copyright 2013}

Kalani et al. This is an open access article distributed under the terms of the Creative Commons Attribution License CC-BY 3.0., which permits unrestricted use, distribution, and reproduction in any medium, provided the original author and source are credited. need to traverse a larger distance of bone. Figure 1 illustrates the axial and sagittal trajectory difference between transpedicular (TP) and transfacet (TF) screw placement. 


\section{Cureus}
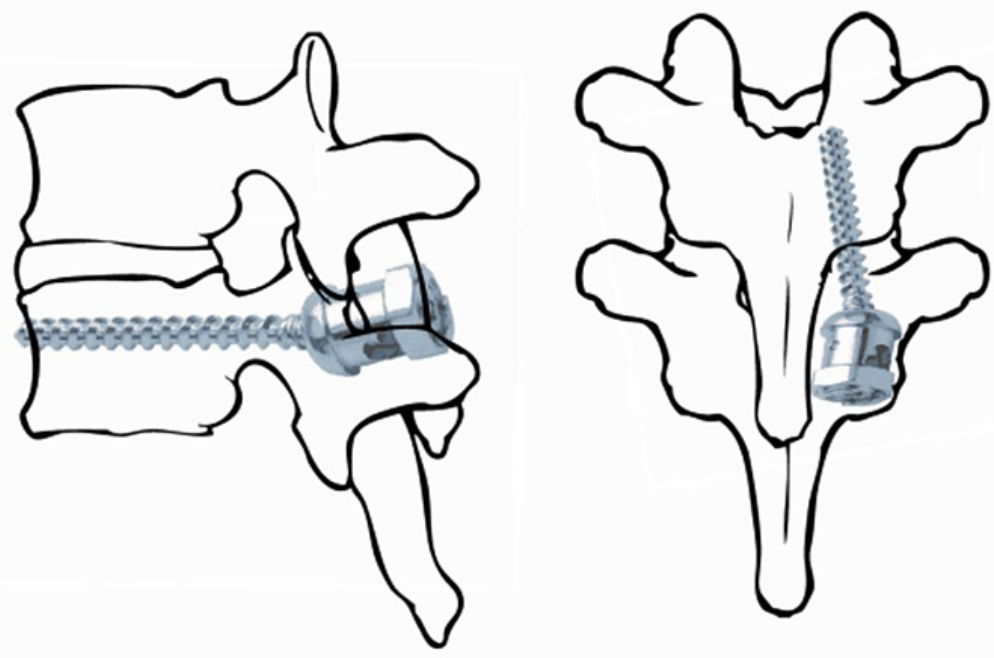

$1 \mathrm{~A}$
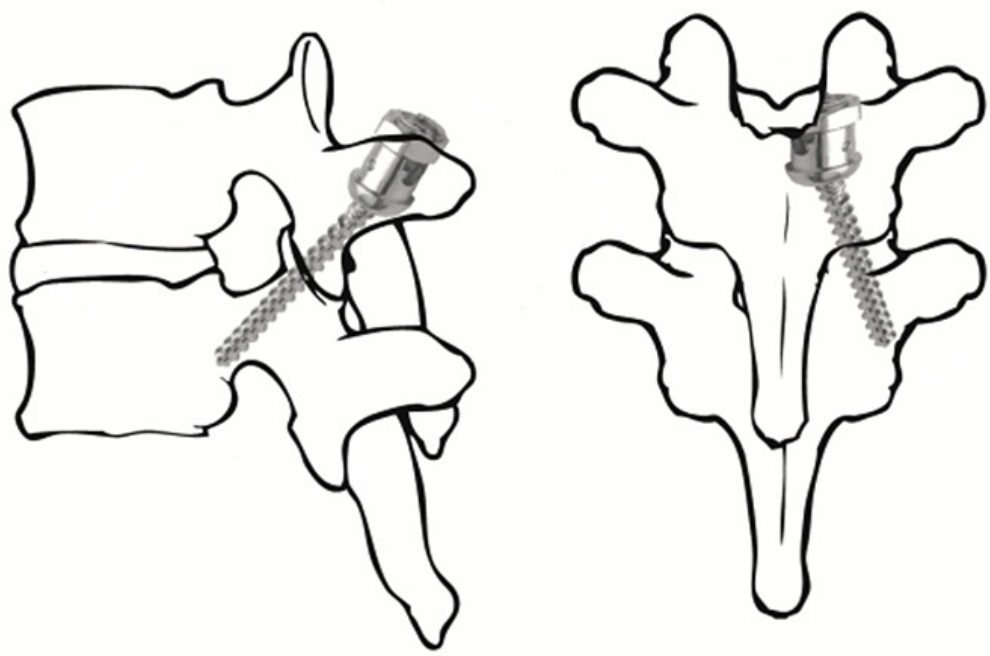

$1 B$

\section{FIGURE 1: Trajectory Diagrams}

IA. Trajectory of Transpedicular Screw Fixation. IB. Trajectory of Transfacet Screw Fixation

Despite the minimally invasive nature of transfacet screw fixation, this method lost ground to pedicle screw fixation during the 1980s due to its biomechanical inferiority [4]. Pedicle screws are associated with lower incidence of failure at the fusion interface; facet screw failure rates are anecdotally estimated to be higher with repetitive cycling [5]. The difference in biomechanical instability and failure rates have limited the use of facet screws despite similar pseudoarthroses rates between the two methods [1, 2, 6-8]. The use of facet screw fixation has been limited to cases where an interbody buttress - such as a strut or cage - is used to provide added arthrodesis. 
Pedicle screw placement at S1, especially in an obese patient or in patients with difficult sacral anatomy, is technically more difficult compared to transfacet screw placement, however. The higher possibility of screw malposition and associated risks of neurological and vascular damage make their use more difficult compared to facet screw placement. Additionally, the mobilization of paraspinous muscles and the creation of devitalized tissue adds to the increased morbidity of this approach [5, 9]. Moreover, the difficult angulation necessary for the placement of TP screws may require additional incisions, which may increase the risks of infection. Facet screw fixation, on the other hand, provides a relatively less-invasive and equally efficacious, 92\% (TF screw) vs. >90\% (TP screw), approach for posterolateral fusion in a similar manner to pedicle screw fixation [5].

Recently, there has been an increase in the popularity of performing ALIF followed by minimally invasive facet screw fixation posteriorly in patients with degenerative spinal disease with no need for posterior decompression. Facet screw fixation in combination with ALIF has a reported fusion rate of greater than $>95 \%$ [5]. Figure 2 demonstrates postoperative imaging from a patient who underwent L4-5, L5-S1 ALIF followed by minimally invasive L4-5 L5-S1 facet screws placed [7]. In this report, we present a patient who underwent L5-S1 ALIF followed by posterior L5-S1 fusion. We initially planned on placing pedicle screws at L5 and S1, however due to the patient's large bilateral iliac crests, the typical medial trajectory was difficult, requiring a combination of both pedicle screw and facet screw fixation as an alternative trajectory for screw placement. This approach affords 4-cortical purchase of the transfacet screw and can be used in obese patients, patients with hypertrophic iliac crests and those with unfavorable sacral angles. 


\section{Cureus}

\section{FIGURE 2: Postoperative Computed Tomography}

CT scout image demonstrating classic L4/5 and L5/S1 ALIF with posterior L4/5 and L5/S1 facet screws

\section{Technical Report}

\section{History and physical examination}

We evaluated a 50-year-old woman who presented with lower back pain radiating to the bilateral lower extremities. She has previously undergone two L5-S1 laminoforaminotomies and discectomies at an outside hospital. Her prior operations were initially efficacious in ameliorating the pain, but her pain eventually recurred. Steroid injections were attempted but were complicated by epidural hematoma. Reclining ameliorates her pain, but sitting, lying, standing, walking, leaning forward or backward, and twisting all elicit and worsen the pain.

On neurological examination she was alert and oriented, conversant with fluent speech. Cranial nerves, 2-12, were intact. She had full strength in her bilateral upper extremities. Her 
bilateral lower extremities were $4 / 5$ strength in her left hip flexion and left dorsiflexion. She was otherwise $4+/ 5$ strength in her bilateral lower extremities. Sensation was intact to light touch in her bilateral upper and lower extremities and proprioception was intact.

\section{Radiographic imaging}

Preoperative magnetic resonance imaging (MRI) of the lumbar spine, revealed post-surgical changes at L5-S1, with postoperative fluid and granulation tissue in that region (Figure 3). There was a broad based, bulging disk at L5-S1 with encroachment upon the neural foramina, contacting the exiting nerve roots bilaterally, right greater than left, with a 5-mm central disk protrusion.
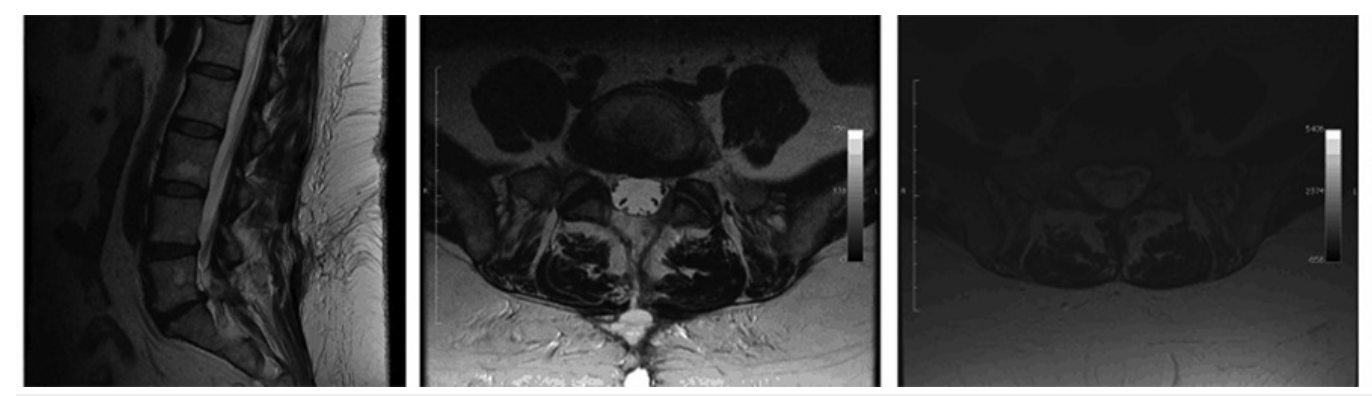

\section{FIGURE 3: Preoperative MRI}

T2 sagittal weighted images of the lumbosacral spine demonstrating post-operative changes from previous laminectomies as well as a symptomatic disc bulge at L5-S1. Note that difficult iliac angles (c) make for technically difficult pedicle screw placement

\section{Surgical procedure}

Our vascular surgery colleagues exposed the anterior L5-S1 disk space through a retroperitoneal approach. After exposure of the L5-S1 level, we utilized intraoperative fluoroscopy to localize the level and proceeded to performing a L5-S1 discectomy and prepared the endplates. A cage was then placed into the disk space and packed with morselized bone allograft and bone morphogenic protein. A buttress plate and screw were placed in the sacral promontory to prevent cage displacement of the construct, which was confirmed with intraoperative fluoroscopy. After completion of the ALIF, the Vascular Surgeons closed the abdominal incision. The patient was repositioned in the prone position.

Once prone, the previous midline incision was identified and re-opened. Missing lamina at the L5 level from her previous laminectomy was identified. Scar dissection was performed followed by re-do laminectomy for decompression of the canal. Concomitantly, we exposed the remainder of the L5 lamina as well as the L5-S1 facet articulation.

Initially, our plan was to perform posterior fixation at L5-S1 using pedicle screws. Because of the patient's narrow iliac window and significant obesity, it was difficult to obtain a suitable trajectory and angle needed to place a satisfactory S1 pedicle screw from a lateral to medial trajectory. Therefore, we decided to place the polyaxial pedicle screw through the facet joint in a transfacet trajectory (medial to lateral). First, we guided the Jamshidi needle through the facet of the L5-S1 in a medial to lateral trajectory into the pedicle of S1 in the direction of the ala and proceeded to place a $35 \mathrm{~mm}$ depth polyaxial screw through the transfacet direction into the ala of the S1 pedicle. Satisfactory placement of the polyaxial transalar-transfacet screw was determined by intraoperative fluoroscopy. Next, a standard pedicle screw was placed at the L5 level at a trajectory of 20 degrees and parallel to the plate with a pedicle finder going through 


\section{Cureus}

the pedicle with a satisfactory cannulation; placement was immediately confirmed with intraoperative fluoroscopy. Titanium rods were placed to secure the two screws affixed to L5 and S1 levels. After satisfactory instrumented stabilization was achieved, morselized bone allograft was added in the posterolateral fashion to achieve the posterolateral fusion. The wound was then closed in standard fashion.

\section{Follow-up}

Postoperative films demonstrate appropriate placement of the anterior construct as well as L5 pedicle and L5-S1 medial-to-lateral facet screw (Figure 4). The patient was seen four weeks after discharge for postoperative follow up. She subjectively stated that her pain had improved "98\% better". Her radicular pain had completely resolved.

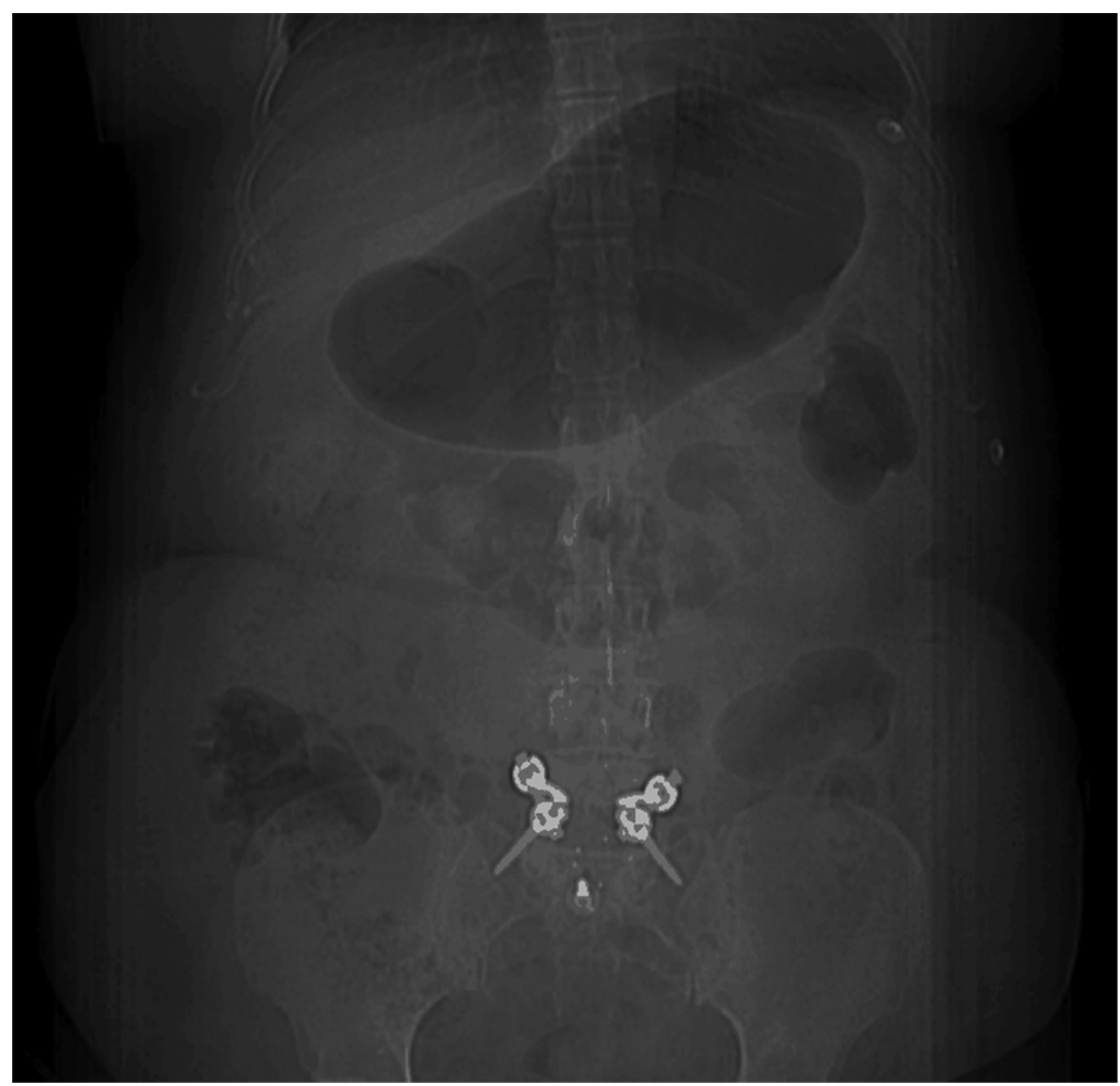

FIGURE 4: Postoperative Computed Tomography.

CT scout image demonstrating L5 pedicle and L5/S1 facet screw as well as ALIF constructs.

\section{Discussion}

Here we demonstrate the use of medial-to-lateral L5-S1 facet fixation with a polyaxial screw, in combination with a L5 pedicle screw, affording greater stabilization than a standard L5-S1 pedicle screw construct in a patient with obesity and unfavorable sacral angles undergoing ALIF. This technique can be utilized for difficult screw placement at S1 in individuals with 
narrow iliac windows. Instead of exposing laterally to find the S1 pedicle where a larger incision may be required, medial-to-lateral fixation at S1 may be achievable in those with hypertrophic iliac crests and unfavorable sacral angles. The method provides less morbidity in patients with said pathology and greater ease for the surgeon.

\section{Conclusions}

Degenerative disc disease is the most common etiology of lower radicular back pain, and is often co-morbid with obesity. In order to obtain appropriate surgical exposure of the S1 pedicle for transpedicular screw fixation, one of two options is available. First, one may extend the incision to allow greater retraction and thus exposure of the pedicle. The other approach would involve a lateral stab incision and percutaneous placement of the pedicle screw. Extension of the incision would increase the invasiveness of the procedure requiring greater paraspinous muscle retraction. In both cases, the patient is faced with a greater likelihood of infection due to larger incision or two incisions that must heal. Once the pedicle is cannulated with the screw, the surgeon is still faced with the task of introducing fixation rods, which may be challenging.

In our patient, due to her narrow iliac window created by large bilateral iliac crests the traditional lateral-to-medial pedicle screw fixation was technically challenging (Figure 3). Of note, this narrow window is seen in large males as well with similar anatomical architecture. We, therefore, placed a traditional L5 pedicle screw and then placed a transfacet screw at L5-S1 in a medial to lateral direction. This screw crossed four cortical bone layers and therefore has sufficient strength. The titanium rod was then connected between the L5 pedicle screw and L5S1 transfacet screw.

The medial-to-lateral approach of the S1 screw demonstrates greater cortical purchase compared to standard S1 pedicle screw fixation (Figure $1 B$ ). There is a risk of neural damage to the lumbosacral plexus, but due to the large territory covered by this screw, the risk of malposition is low. The combination of greater stability, medial to lateral cannulation, and smaller incision all serve to make this approach appropriate for posterior L5-S1 fusion especially in patients with morbid obesity or difficult anatomy.

\section{Additional Information}

\section{Disclosures}

Human subjects: All authors have confirmed that this study did not involve human participants or tissue. Animal subjects: All authors have confirmed that this study did not involve animal subjects or tissue. Conflicts of interest: In compliance with the ICMJE uniform disclosure form, all authors declare the following: Payment/services info: All authors have declared that no financial support was received from any organization for the submitted work. Financial relationships: All authors have declared that they have no financial relationships at present or within the previous three years with any organizations that might have an interest in the submitted work. Other relationships: All authors have declared that there are no other relationships or activities that could appear to have influenced the submitted work.

\section{References}

1. Boucher HH: Method of spinal fusion. Clin Orthop Relat Res. 1997, 335:4-9.

2. King D: Internal fixation for lumbosacral fusion. J Bone Joint Surg Am. 1948, 30A:560-565.

3. Magerl FP : Stabilization of the lower thoracic and lumbar spine with external skeletal fixation. Clin Orthop Relat Res. 1984, 189:125-141. http://dx.doi.org/10.1097/00003086198410000-00014

4. Vaccaro AR, Garfin SR: Pedicle-Screw Fixation in the Lumbar Spine . J Am Acad Orthop Surg. 1995, 3:263-274. 


\section{Cureus}

5. McGirt MJ, Sutter EG, Xu R, Sciubba DM, Wolinsky JP, Witham TF, et al,: Biomechanical comparison of translaminar versus pedicle screws at T1 and $\mathrm{T} 2$ in long subaxial cervical constructs. Neurosurgery. 2009, 65:167-172. 10.1227/01.NEU.0000345642.50726.A3

6. El Masry MA, McAllen CJ, Weatherley CR: Lumbosacral fusion using the Boucher technique in combination with a posterolateral bone graft. Eur Spine J.. 2003, 12:408-412. 10.1007/s00586003-0566-3

7. Jang JS, Lee SH: Clinical analysis of percutaneous facet screw fixation after anterior lumbar interbody fusion. J Neurosurg Spine . 2005, 3:40-46. 10.3171/spi.2005.3.1.0040

8. Margulies JY, Seimon LP: Clinical efficacy of lumbar and lumbosacral fusion using the Boucher facet screw fixation technique. Bull Hosp Jt Dis. 2000, 59:33-39.

9. Ferrara LA, Secor JL, Jin BH, Wakefield A, Inceoglu S, Benzel EC: A biomechanical comparison of facet screw fixation and pedicle screw fixation: effects of short-term and long-term repetitive cycling. Spine (Phila Pa 1976). 2003, 28:1226-1234.

10.1097/01.BRS.0000065485.46539.17 